\title{
Effect of Interface on the Deformation of Aluminium Bicrystal: Atomistic Simulation Study
}

\author{
Shaohua Yan, Bobin Xing and Qing-Hua Qin ${ }^{a}$ \\ Research School of Engineering, The Australia National University, Acton, ACT 2601, Australia
}

\begin{abstract}
Molecular dynamic (MD) simulation has been conducted to study the effect of interface structure on the mechanical response of eight $<110>$ symmetric tilt grain boundaries in high stacking-fault Al. It is found that the grain boundaries with E structure unit (SU) have higher energy, but the grain boundary energy alone cannot be used as a parameter to determine the mechanical properties of grain boundary. The SUs, especially E units, do have an influence on the mechanical response of grain boundaries. Our results show that the dislocation imitates from $\mathrm{E}$ units preferably, but this depends on the dissociation at grain boundary.
\end{abstract}

\section{Introduction}

In the last decade, experiment of single crystals at micron/submicron scale length has been extensively studied. One of the well-known conclusions from these microscale tests is the relation between the sample size and flow stress, which can be described as the below power-law:

$$
\sigma \propto D^{-} n
$$

Where $\sigma$ is the flow stress, $\mathrm{D}$ presents the diameter of the sample, and $\mathrm{n}$ is the fitting value. Unlike the deformation in the metallic foil [1-4], the deformation of single crystal at micon/submicron scale is featured with [5]: (1) a dramatic increase and stochastic variation of flow stress with decreasing sample size, (2) intermittency in plastic flow and strain burst (avalanches), (3) strength and avalanche properties that depend on the initial dislocation density. Up to date, most of the research has focused on the deformation of single crystals. However, most of real engineering materials are polycrystalline or multiphase. It is reported that the interface has had an influence on the strength of materials, which depends on the type of the interface. Allison Kunz et al. [6] reported that the single vertical grain boundary of pure aluminium reduce the degree of strain hardening and causes lower flow stress, while $\mathrm{Ng}$ and Ngan [7] reported the presence of grain boundary leads to increasing both the strain-hardening rate and flow stress. The difference is probably due to the structure of grain boundaries. As suggested by Ref. [8], the deformation/dislocation emission is largely depended on the atomistic structure of grain boundaries. For experiment, it is unrealistic to explore the relationship between interface structure and deformation mechanism systematically. Simulation may give us possible path to get insight into the plasticity of nanomaterials at submicron/submicron length.

In this paper, molecular dynamics is used to study the effect of interface structure on the deformation of eight $<110>$ symmetric tilt grain boundaries in high stacking-fault aluminium. First,

\footnotetext{
${ }^{\mathrm{a}}$ Corresponding author: qinghua.qin@anu.edu.au
} 
the grain boundary (GB) structure at equilibrium condition is identified using structure unit theory. Then uniaxial tensile deformation is applied to GBs with minimum energy. Finally, the dislocation nucleation is then discussed to get insight into the GB structure's influence.

\section{Methodology}

The computational model used in this study is a bicrystal with a grain boundary located in the centre, as shown in Fig.1, all Al bicrystals used in this research have a symmetric tilt misorientation about [110] axis, which is z-axis in this research. The interface misorientation angle is measured used the [001] direction as the reference $\left(0^{\circ}\right)$. As shown in table 1, eight different GBs with different misorientation angles have been simulated.

In order to get GB structures with minimum energy, different initial GB structures have been achieved in two ways: (1) changing the initial distance between grain $\mathrm{A}$ and grain $\mathrm{B}$, in which nine

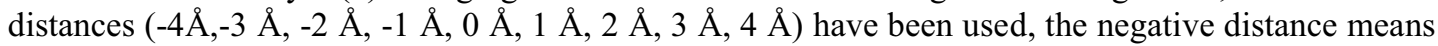
that there is an overlap between these two grains; (2) altering the cut-off regime, which arranges from $0.25 \mathrm{a}_{0}$ to $0.7 \mathrm{a}_{0}$ with an increment of $0.05 \mathrm{a}_{0}$, where $\mathrm{a}_{0}$ is the lattice constant. Periodic boundary conditions are used for all directions when finding the minimum energy.

For this work, all the simulation is conducted using LAMMPS [9]. The embedded-atom method (EAM) potential is developed by Mishin [10]. After the minimum energy configuration is attained, the simulation model is equilibrated using MD in the isobaric-isothermal (NPT) ensemble at a pressure of 0 bar and a temperature of $10 \mathrm{~K}$ for $20 \mathrm{ps}$. A constant rate of $1 \times 10^{9} \mathrm{~s}^{-1}$ is applied perpendicular to the boundary plane (along Y direction) at a temperature of $10 \mathrm{~K}$. The visualization tools Ovito [11] are used to illustrate of the bicrystal models. The common neighbour analysis (CNA) [12] technique is used to identify the defect structure and its evolution during the simulations. The dislocation extraction algorithm (DXA) [13] is used to convert identified dislocations into continuous lines and compute their Burgers vectors.

Table 1. The misorientation angles, grain boundary plane normal, dimensions, and number of atoms for the ten symmetric boundaries in this study.

\begin{tabular}{cccccc}
\hline $\begin{array}{c}\text { GB plane normal } \\
\text { and CSL } \\
\text { designation }\end{array}$ & $\begin{array}{c}\text { Misorientation } \\
\text { angle } \theta\left(^{\circ}\right)\end{array}$ & SU period & $\begin{array}{c}\text { Calculated GB } \\
\text { energy }\left(\mathrm{mJ} / \mathrm{m}^{2}\right)\end{array}$ & $\begin{array}{c}\text { Dimensions } \\
\mathrm{X} \times \mathrm{Y} \times \mathrm{Z}(\mathrm{nm})\end{array}$ & Atoms \\
\hline$\sum 33(554)$ & 121.0 & |DDE.DDE| & 319 & $16.29 \times 32.9 \times 4$ & 128870 \\
$\sum 11(332)$ & 129.5 & |DE.DE| & 411 & $17.46 \times 34.2 \times 4$ & 143962 \\
$\sum 9(221)$ & 141.1 & $\mid$ E.El & 451 & $17.18 \times 34 \times 4$ & 140832 \\
$\sum 19(331)$ & 153.5 & $\mid$ EA.EA| & 430 & $17.48 \times 35.3 \times 4$ & 148568 \\
$\sum 33(441)$ & 160.0 & $\mid$ |EAA.EAA| & 417 & $16.45 \times 32.58 \times 4$ & 128520 \\
$\sum 73(661)$ & 166.6 & $\left|E^{\prime} A^{\prime} A^{\prime} . E^{\prime} A^{\prime} A^{\prime}\right|$ & 363 & $14.68 \times 34.6 \times 4$ & 121716 \\
$\sum 9(114)$ & 38.9 & |BC.BC| & 330 & $17.01 \times 34.36 \times 4$ & 140532 \\
$\sum 11(113)$ & 50.5 & $\mid$ C.Cl & 150 & $17.10 \times 34.92 \times 4$ & 143640 \\
\hline
\end{tabular}

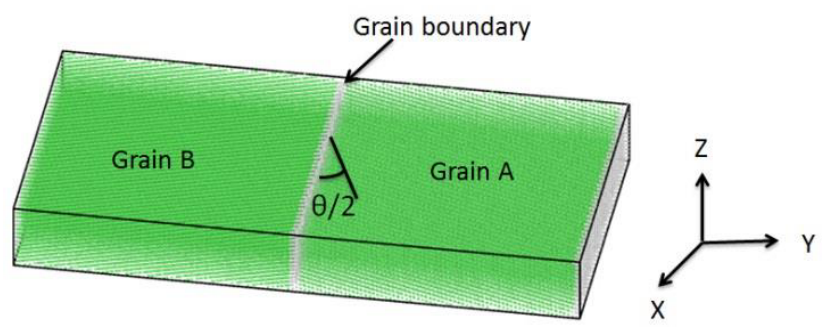

Figure 1. Schematic of Al bicrystal interface model. The interface is generated by a symmetric tilt rotation of grain $\mathrm{A}$ and grain $\mathrm{B}$ around [110] axis. 


\section{Results and discussion}

\subsection{GB structure of the Al bicrystal and energy}

The GB structures with minimum energy after calculating are shown in Fig.2 (a) $\sim(\mathrm{h})$. The centrosymmetric parameter was used to identify the structure units (SUs) that were used to identify the atoms associated with the GB. In this paper, the definition of SUs is similar to Ref. [14]. For the misorientation angle ranging from $121^{\circ}$ to $141.1^{\circ}$, as shown in Fig.2 (a) (c), there are two primary SUs found in these GB, which are D SU and E SU. Besides, in this misorientation angle range $\left(121.0^{\circ}<\theta<141.1^{\circ}\right)$, the E SU approaches each other when $\theta$ ranges from $121^{\circ}$ to $141.1^{\circ}$, there is only E SU for $\sum 9(221)$. The GB structure with $\theta>141.1^{\circ}$ are also characterized with two primary SUs, A (A') unit and $\mathrm{E}\left(\mathrm{E}^{\prime}\right)$ unit, and the distance between adjacent units increases as well. For the equilibrium GB structure of $\sum 9$ (114) and $\sum 11$ (113), C and B units are the two SUs. From Table 1, it can be seen that the GB energy is various with respect to misorientation angle. However, GBs with E/E' SU, such as $\sum 9(221)$ and $\sum 19(331)$, are found to have higher energy because this SU leads to pronounced GB expansion to accommodate large free volumes [15]. It is also reported that the presence of E SU would reduce the strength of the interface $[15,16]$. The influence of these GB structures on the mechanical response will be discussed later in this paper.
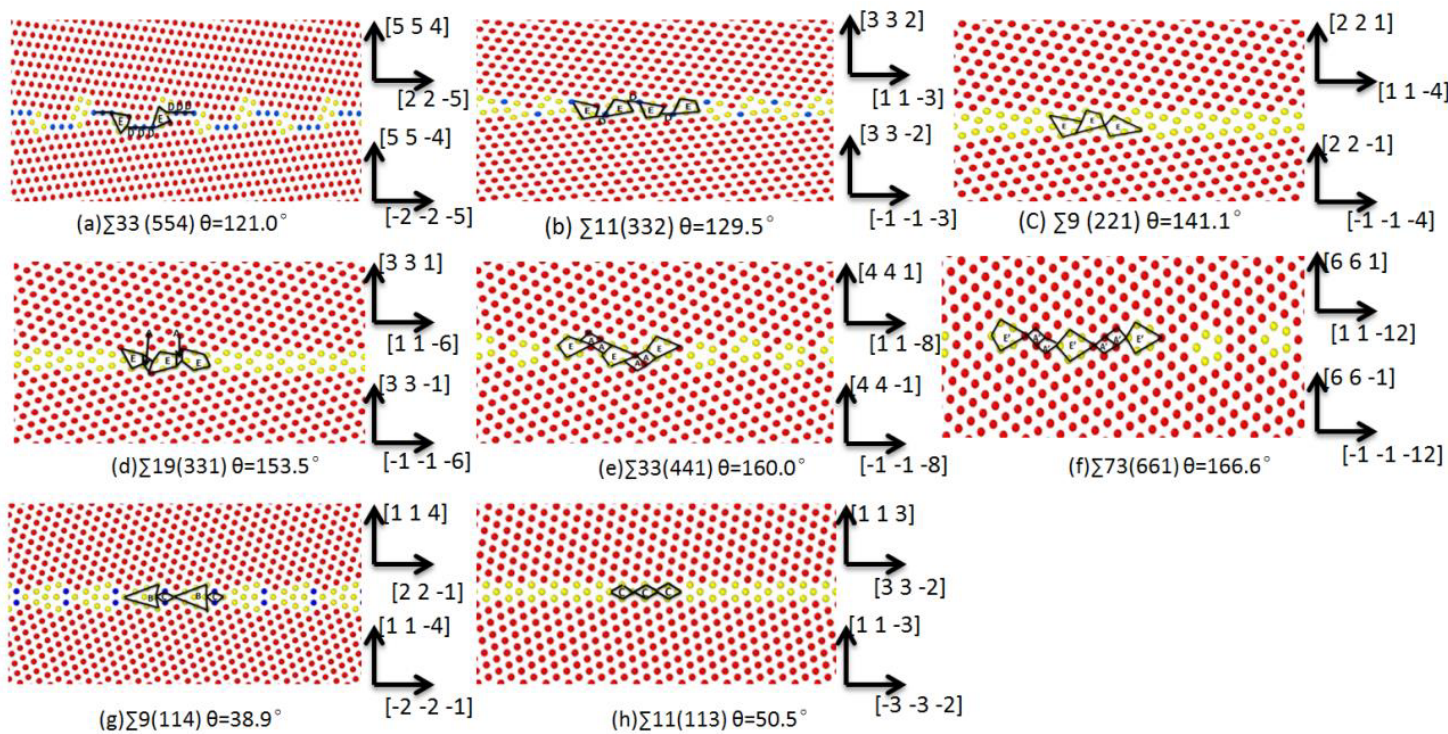

Figure 2. Eight $\mathrm{GB}$ structures in $\mathrm{Al}$ for various misorientation angle $\theta$ at $0 \mathrm{~K}$. The structures are view along [110] tilt axis. Images are coloured according to CNA parameters. Atoms with red colour are perfect fec structures, while yellow atoms stand for GB and dislocation, and blue atoms represent the stacking fault.

\subsection{Stress-Strain behaviour of the Al bicrystal}

The uniaxial tensile stress-strain behaviour of Al bicrystals is shown in Fig.3. The results of GB without E SU are represented in Fig.3 (a), while the results of GB with E SU are displayed in Fig.3 (b). Compared with GB without E SU, the maximum tensile stress and the corresponding strain for GBs with E SU are relatively smaller. Fig.4 shows the stress required for the dislocation nucleation for the eight $<110>$ tilt grain boundaries. It can be seen the stress for GBs with E unit is lower than those without E unit. Recall that GBs with E SU have higher energy than those without E SU, it seems the GB energy can be used as a parameter to determine the mechanical properties of GB. However, for 
the GB with E SU, the lowest stress is located in $\sum 33$ (554) $\theta=121^{\circ}$, which has lowest GB energy among GBs with $\mathrm{E}$ unit. The highest energy grain boundary, $\sum 9(221)$, represents higher stress than these GBs with lower energy, which are $\sum 33$ (554), $\sum 11$ (332), and $\sum 73(661)$. Thus, grain boundary energy alone cannot be used to determine the mechanical properties of GB, the GB structure should also be taken into account because our results imply that the GB structure can have an influence on the mechanical properties of the interface.

Tucker et al. [16] also reported that the E SU has an effect on the properties of grain boundaries of $\mathrm{Cu}$ bicrystal. They found that the more ratio of E SU existing in the GB structure, the smaller stress required for the dislocation nucleation. Thus, for their case, the smallest stress was found in $\sum$ 9(221), in which only E SU is found at the GB. Same results can also be found in Ref.[15], where $\sum$ 9(221) of $\mathrm{Al}$ and $\mathrm{Cu}$ displays smallest shear strength, but the SUs at $\sum 9(221)$ in Ref. [15] are C unit and B unit. Nonetheless, for our case, the smallest one is the $\sum 33(554) / \theta=121^{\circ}$. This discrepancy can be interpreted by the following two facts: (1) Al has higher stacking fault than $\mathrm{Cu}$, which may differentiate the mechanical behaviour, (2) different EAM potentials used for Al, which cause completely different SUs for the same $\sum$ tilt grain boundary, thus the mechanical response is changed accordingly.
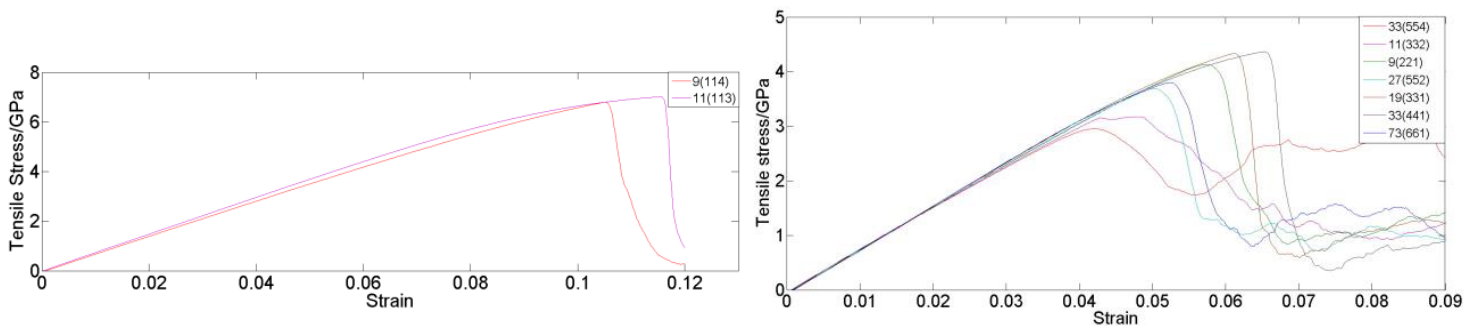

Figure 3. Stress-Strain response of the Al bicrystal at 10K, (a) without E unit, (b) with E unit

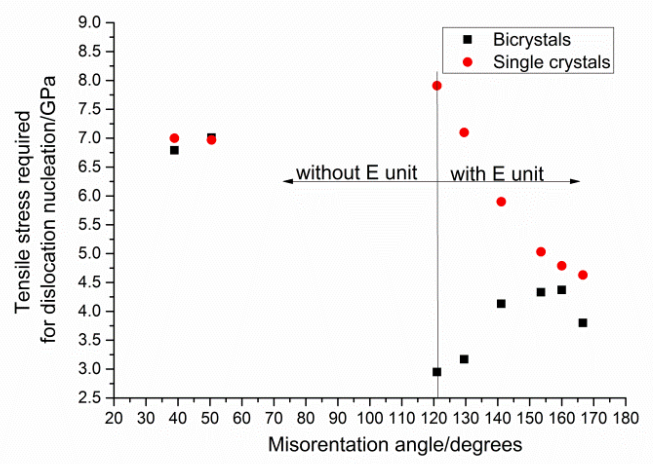

Figure 4. Tensile stress required for dislocation nucleation for the eight symmetric tilt grain boundaries.

\subsection{Dislocation nucleation of the Al bicrystal}

From the section 3.2, it can be found that the SU plays a key role on mechanical behaviour of the Al bicrystal. To get insight into the mechanism of the dislocation, visual inspection of the MD simulation is conducted using Ovito, the results are shown in Fig. 5 and Fig.6. Fig.5 shows the dislocation nucleation and propagation of two GB with E SU. For $\sum 9(221)$, dislocation nucleates from the upper and lower grain boundaries, and the free surface. Until $\varepsilon=0.062$, there are some partial dislocations growing from the middle grain boundary. But for $\sum 33(554)$, the dislocation starts from the grain 
boundary, including the middle grain boundary. More specifically, the dislocation initiates from $\mathrm{E}$ units. Comparing the SU period of these two grain boundaries, SU period of $\sum 9(221)$ consists of $\mid$ E.El, while $\sum 33(554)$ of IDDDE.DDDEl. It seems the dissociation at GB can trigger the emission of dislocation from E SU. Fig.6 illustrates the dislocation behaviour of the Al bicrystal without E SU. For $\sum$ 11(113) with the SU period $\mid \mathrm{C} . \mathrm{Cl}$, as shown in Fig.6(a), the dislocation initiates from the free surface, and dislocation can be found on the grain boundary only after the strain reaches 0.117 . As for $\sum 9$ (114) with the SU period $|\mathrm{BC} . \mathrm{BC}|$, the dislocation nucleates from the grain boundary at smaller strain $(\varepsilon=0.106)$. Similar to the GB with E SU, the dissociation at GB without E SU seems to trigger the emission of dislocation as well.

(a)

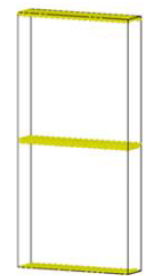

$\sum 9(221), \varepsilon=0.0$

(b)

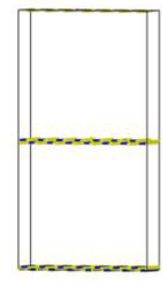

$\sum 33(554), \varepsilon=0.0$

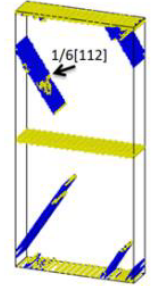

$\sum 9(221), \varepsilon=0.06$

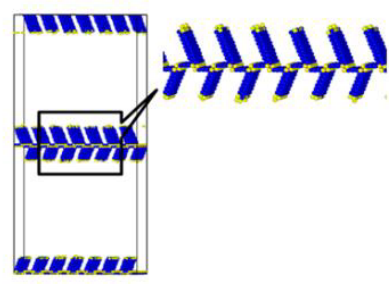

$\sum 33(554), \varepsilon=0.044$

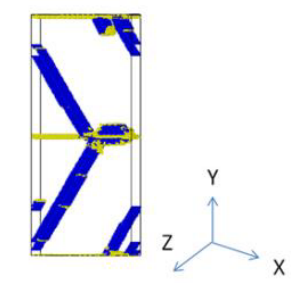

$\sum 9(221), \varepsilon=0.062$

Figure 5. Snapshot of Al bicrystal with E SU GB at different deformation stage. (a) dislocation nucleation and propagation of $\sum 9(221)$ with the SU period IE.El, (b) dislocation nucleation and propagation of $\sum 33(554)$ with the SU period IDDDE.DDDEl. Images are coloured according to CNA parameters. Atoms with red colour are perfect fcc structures, while yellow atoms stand for GB and dislocation, and blue atoms represent the stacking fault.

(a)

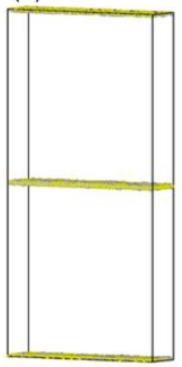

$\sum 11(113), \varepsilon=0.0$

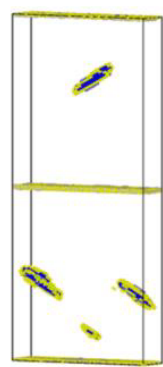

$\sum 11(113), \varepsilon=0.116$

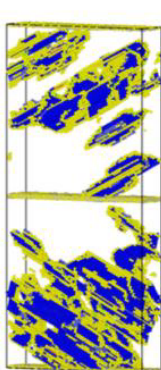

$\sum 11(113), \varepsilon=0.117$

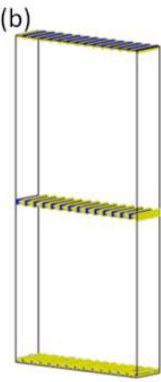

$\sum 9(114), \varepsilon=0.0$

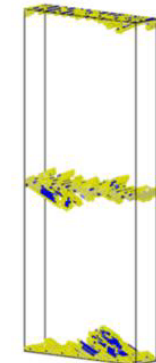

$\sum 9(114), \varepsilon=0.106$

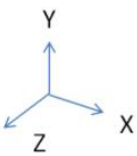
Figure 6. Snapshots of Al bicrystal without E SU GB at different deformation stages. (a) Dislocation nucleation
and propagation of $\sum 11(113)$ with the SU period $\mid \mathrm{C} . \mathrm{Cl}$, (b) Dislocation nucleation and propagation of $\sum 9$ (114) with the SU period $\mid \mathrm{BC} . \mathrm{BCl}$. Images are coloured according to CNA parameters. Atoms with red colour are perfect fcc structures, while yellow atoms stand for GB and dislocation, and blue atoms represent the stacking fault. 


\section{Conclusions}

In this paper, MD simulation has been performed to study the influence of interface structure on the mechanical properties of $<110>$ symmetric tilt grain boundaries in Al. Our results show that GBs with E SU have higher energy, but the GB energy alone cannot be used as a parameter to determine the mechanical properties of GB. The dissociation at GB also has an effect on the deformation behaviour of grain boundaries. When there is dissociation at GB structure, the dislocation prefers to initiate from E SU, while dislocation nucleates from surface atoms when there is no dissociation at GB structure.

\section{ACKNOWLEDGEMENT}

The MD simulation was conducted using NCI National Facility of Australia. The author, Shaohua Yan, would like to acknowledge the financial support from China Scholarship Council (CSC). This work was financially supported from Australian Research Council under Linkage project (grant no. LP130101001).

\section{References}

[1] Kang YL, Zhang ZF, Wang HW, Qin QH. Mater. Sci. Eng., A 394, 312 (2005).

[2] Kang YL, Wang HW, Zhang ZF, Qin QH. Microscopic Experimental Analysis of Fracture Toughness in Fracture of Copper Foils. Proc Of 11th International Conference on Fracture (in CD-ROM, ISBN: 9788890318818), (2005).

[3] Kang YL, Zhang ZF, Wang HW, Qin QH. Experimental investigations of size effect on fracture toughness of metallic foils, in: ECF15, Stockolm (2004).

[4] Wang HW, Kang YL, Zhang ZF, Qin QH. Int. J. Frac. 123, 177 (2003).

[5] Uchic MD, Shade PA, Dimiduk DM. Annu. Rev. Mater. Sci. 39, 361 (2009).

[6] Kunz A, Pathak S, Greer JR. Acta Mater. 59, 4416 (2011).

[7] Ng KS, Ngan AHW. Philos. Mag. A 89, 3013 (2009).

[8] Spearot D, Tschopp M, Jacob K, McDowell D. Acta Mater. 55, 705 (2007).

[9] Plimpton S. J. Comput. Phys. 117, 1 (1995).

[10] Mishin DF. Phys. Rev. B 59, 3393 (1999).

[11] Stukowski A. Modell. Simul. Mater. Sci. Eng. 18, 015012 (2010).

[12] Schiøtz J, Di Tolla FD, Jacobsen KW. Nature 391, 561 (1998).

[13] Stukowski A, Albe K. Modell. Simul. Mater. Sci. Eng. 18, 085001 (2010).

[14] Rittner J, Seidman D. Phys. Rev. B 54, 6999 (1996).

[15] Sansoz F, Molinari JF. Acta Mater. 53, 1931 (2005).

[16] Tucker GJ, Tschopp MA, McDowell DL. Acta Mater. 58, 6464 (2010). 